\title{
Uma nova espécie de Arlea Womersley do sudeste do Brasil (Collembola, Isotomidae)
}

\author{
Eduardo A. Abrantes \& Maria C. de Mendonça
}

${ }^{1}$ Departamento de Entomologia, Museu Nacional, Universidade Federal do Rio de Janeiro. 20940-040 Rio de Janeiro, Rio de Janeiro,Brasil.E-mail: mcleide@acd.ufrj.br; eassis@mn.ufrj.br

\begin{abstract}
A new species of Arlea Womersley from southeastern Brazil (Collembola, Isotomidae). Arlea arenicola sp. nov. is described and illustrated. It was collected at Restinga de Maricá, Maricá, Rio de Janeiro, Brazil, on sand dunes covered by herbaceous vegetation.
\end{abstract}

KEY WORDS. Arlea arenicola, littoral, restinga.

RESUMO. Arlea arenicolasp. nov. é descrita e ilustrada. Coletada na Restinga de Marica, Marica, Rio de Janeiro, Brasil, em dunas de areia coberta por vegetação herbácea.

PALAVRAS CHAVE. Arlea arenicola, litoral, restinga.

O conhecimento da biodiversidade de Collembola em ecossitema costeiro no Brasil, ainda, é bastante restrito, embora diversos estudos envolvendo Poduromorpha venham sendo realizados nos últimos anos (Mendonça \& Fernandes 1997, Fernandes \& Mendonça 2002, 2004). Durante as investigações sobre a fauna de Isotomidae em áreas de restinga no sudeste brasileiro foi constatada, dentre amplo material, uma nova espécie do gênero Arlea Womersley, 1939. Este gênero é caracterizado pela ausência de pigmento, olhos reduzidos ou ausentes, órgão pós-antenal grande e oval, furca desenvolvida, mucro falciforme e os dois últimos segmentos abdominais fusionados. Arlea está representado, até o momento, por quatro espécies A. lucifuga (Arlé, 1939) e A. spinisetis Mendonça \& Arlé, 1987 (do Brasil), A. caeca Rapoport \& Rubio, 1968 (do Chile) e $A$. tridens Barra, 1997 (da África do Sul).

Arlea arenicola sp. nov. ocorre em dunas cobertas por vegetação herbácea pertencentes aos gêneros Allagoptera Nees (Arecaceae), Erythroxylum P. Browne (Erythroxylaceae) e Manilkara Adans (Sapotaceae).

O material-tipo encontra-se depositado na Coleção de Collembola do Museu Nacional (CM/MNRJ), Universidade Federal do Rio de Janeiro, Rio de Janeiro.

\section{Arlea arenicola sp. nov.} Figs 1-9

Comprimento do corpo variando de 0,45-0,56 $\mathrm{mm}$. Habitus alongado e cilíndrico. Coloração branca. Cerdas da cabeça e dos tergitos lisas, subiguais, algumas um pouco mais longas, como as da parte posterior cefálica, das laterais do tórax e da parte posterior dos tergitos abdominais V-VI (Figs 1 e 2); sensilas de revestimento pequenas, subcilíndricas e subiguais.
Antenas mais longas que a diagonal cefálica (relação antena: cabeça $=1: 0,8)$. Segmento antenal IV provido de microsensila subapical dorso-externa, protegida por pequena cerda curva; seis sensilas espessas e cilíndricas; 2-3 sensilas suplementares muito finas. Órgão sensorial do segmento antenal III constituído por duas microsensilas protegidas por $1+1$ sensila lateral cilíndrica; mais abaixo lateralmente duas sensilas, uma micro e uma robusta. Segmento antenal II com 28 cerdas de tamanhos variados; uma sensila lateral robusta e duas microquetas basais, uma dorsal e uma ventral. Segmento antenal I com 16 cerdas; duas sensilas ventro-distais cilíndricas e de tamanhos desiguais; duas microquetas basais, uma ventral e uma dorsal (Figs 3 e 4); relação dos segmentos antenais I:II:III:IV = $20 \mu \mathrm{m}$ : $25 \mu \mathrm{m}: 25 \mu \mathrm{m}: 47 \mu \mathrm{m}$.

Sem olhos. Órgão pós-antenal grande e oval $(16 \mu \mathrm{m}) \mathrm{com}$ cerca de 8-9 cerdas dispostas inferiormente em semicírculo (Fig. 5). Estrutura labial simples, lobo externo da maxila não individualizado. Segmento torácico II com $8+8$ cerdas axiais, $3+3$ sensilas laterais, sendo duas mais internas e uma maior e mais externa. Segmento torácico III com $5+5$ cerdas axiais, $2+2$ sensilas laterais, a mais externa ligeiramente afilada. Segmentos abdominais I e II respectivamente com $3+3$ cerdas axiais, $1+1$ sensila lateral. Segmento abdominal III com $4+4$ cerdas axiais, $1+1$ sensila lateral. Segmento abdominal IV constituído por $4+4$ cerdas axiais e três cerdas ímpares, $1+1$ sensila lateral. Segmento abdominal V-VI com $2+2$ cerdas axiais, $1+1$ sensila dorso lateral ( $\mathrm{spl}$ ) ligeiramente maior e mais espessa que as demais $(6 \mu \mathrm{m}) ; 1+1$ sensila ventral (sv) $(4 \mu \mathrm{m})$; cerdas ímpares a0, m0, p0, medindo respectivamente $10 \mu \mathrm{m}, 17 \mu \mathrm{m}, 5 \mu \mathrm{m}$ (Fig. 6).

Pernas revestidas de cerdas simples; unha simples, sem dente na crista interna $(10 \mu \mathrm{m})$; apêndice empodial com ápice afilado e lamela arredondada; "tenent hair" tibiotarsal afilado. 


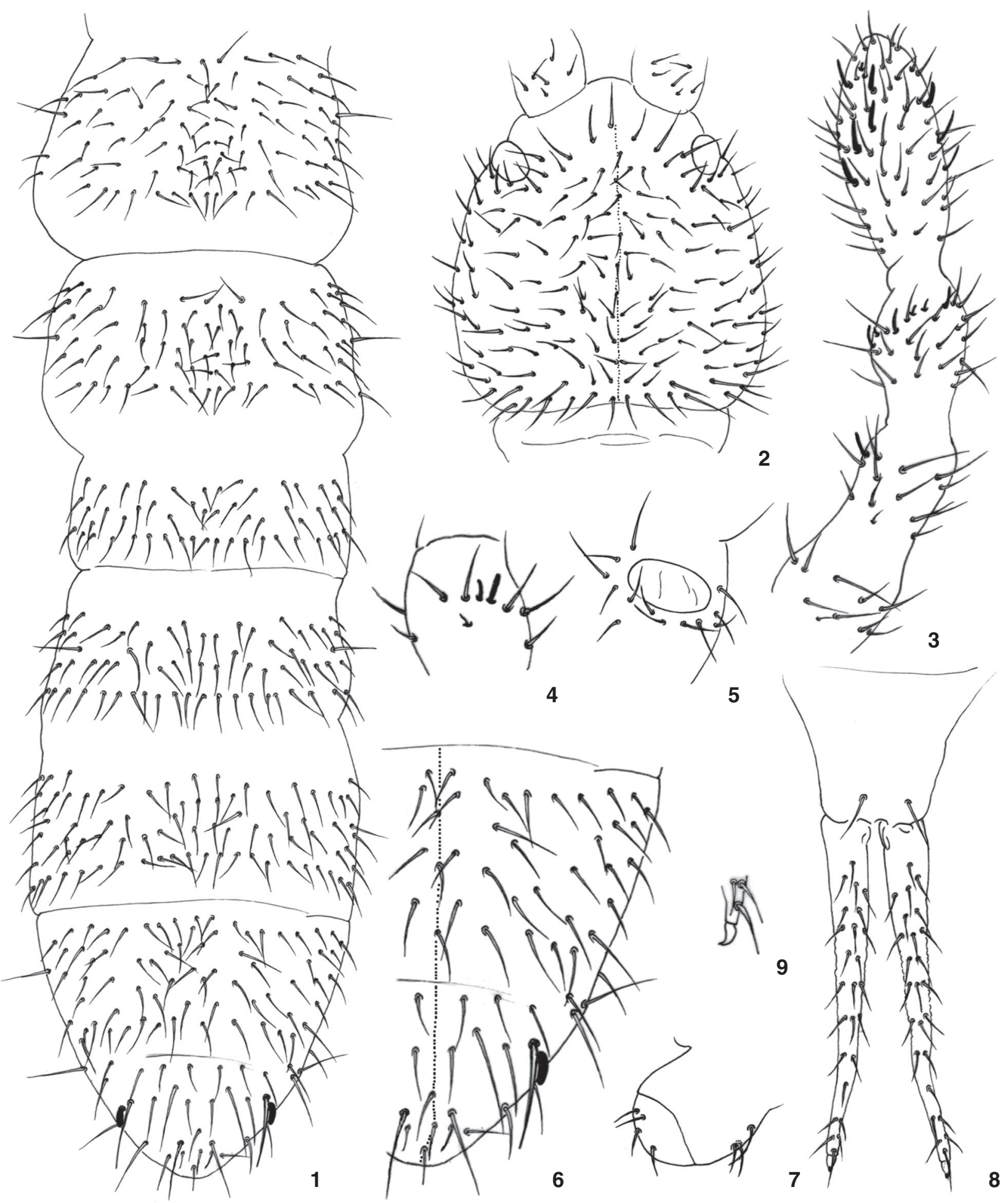

Figuras 1-9. Arlea arenicola sp. nov.: (1) vista dorsal do tórax e abdômen; (2) cabeça, quetotaxia; (3) antena; (4) face ventral do segmento antenal I; (5) órgão pós-antenal; (6) área dorso distal dos tergitos abdominais IV-VI; (7) face lateral do tubo ventral; (8) face ventral da furca; (9) mucro. 


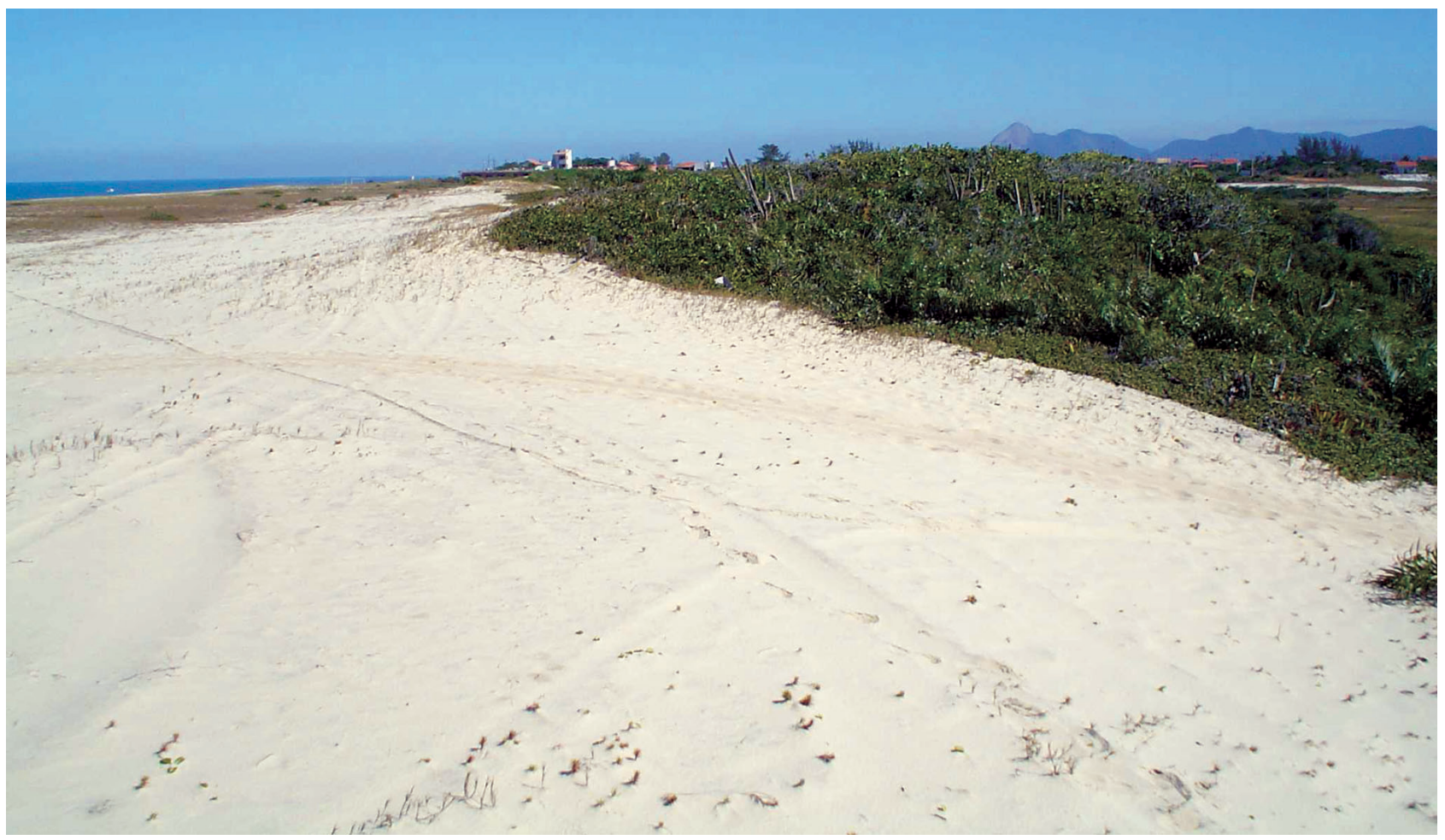

Figura 10. Vista da área das dunas cobertas por vegetação herbácea na Restinga de Maricá, Rio de Janeiro, onde foi coletada A. arenicola sp. nov.

Tubo ventral desprovido de cerdas na face anterior, $4+4$ cerdas distais e $3+3$ posteriores (Fig. 7). Tenáculo com $4+4$ dentes e uma cerda. Subcoxa furcal anterior com 10-14 cerdas e subcoxa furcal posterior com 6-7 cerdas. Furca normalmente desenvolvida e crenulada; manúbrio com $1+1$ cerda na face ventro-distal e 18-24 cerdas dorsais; "dens" com 20-25 cerdas ventrais e 6+6 dorsais; mucro pequeno e falciforme (Figs 8 e 9). Comprimento do manúbrio: dens: mucro = $35 \mu \mathrm{m}: 80 \mu \mathrm{m}: 4 \mu \mathrm{m}$.

Material examinado. Holótipo fêmea. Brasıl, Rio de Janeiro: Maricá, Restinga de Maricá, em dunas cobertas por vegetação herbácea, E.A. Abrantes \& L.H. Fernandes leg. Em lâmina 1506 CM/MNRJ, 15/VII/2004. Parátipos da mesma procedência em lâminas: 1268, 2 exemplares; 1269, 1 ex., 28/I/2003, 1505, 5 exemplares, 1506, 18 exemplares fêmeas e 1 exemplar macho, 15/VII/2004.

Etimologia. arenicola, em alusão ao meio arenoso no qual foi encontrada.

Discussão. Dentre as espécies do gênero Arlea, as que mais se aproximam de $A$. arenicola sp. nov. são $A$. caeca e $A$. tridens, com as quais compartilha a ausência de olhos. Comparando ainda $A$. arenicola sp. nov. e $A$. caeca constatou-se semelhanças relacionadas à ausência de dentes na crista interna da unha e ausência de "tenent hair" espatulado. Entretanto, diferença marcante foi observada quanto ao número de cerdas na furca, uma vez que $A$. arenicola sp. nov. apresentou entre $20-25$ cerdas na dens e apenas $1+1$ cerda ventro-distal no manúbrio, diferentemente daquelas descritas para A.caeca com $34+34$ cerdas dentais e $5+5$ manubriais.

À semelhança de $A$. tridens, encontrada em dunas arenosas na Província de Natal (África do Sul), a nova espécie também foi coletada em dunas arenosas cobertas por vegetação herbácea (Fig. 10). Não obstante as afinidades ambientais dessas espécies, $A$. arenicola sp. nov. se diferencia facilmente de $A$. tridens por um grande número de caracteres morfológicos. Segundo BARRA (1997) a sua espécie africana tem uma estrutura quitinosa com três ramos no lábio que a torna diferente de todas as outras espécies. Além disto, a quetotaxia de revestimento e a sensilar, o número de sensilas no segmento antenal IV, as cerdas do manúbrio e da dens e a estrutura labial em A. arenicola sp. nov. descartam qualquer semelhança com $A$. tridens.

\section{AGRADECIMENTOS}

Agradecemos à colega Liliane Henriques Fernandes, MSc. do Laboratório de Apterygotologia do Departamento de Entomologia do Museu Nacional, UFRJ pela leitura crítica e valiosas sugestões, além do companheirismo e agradável convivência no decorrer dos trabalhos. À Profa Dra. Heloísa Alves de Lima Carvalho pela companhia nas excursões e pelas iden-

Revista Brasileira de Zoologia 22 (4): 936-939, dezembro 2005 
tificações botânicas. À Fundação Carlos Chagas Filho de Amparo a Pesquisa do Estado do Rio de Janeiro (FAPERJ) pela concessão de bolsa de estudo.

\section{REFERÊNCIAS BIBLIOGRÁFICAS}

BARRA, J-A. 1997. Nouveaux Collemboles Entomobryomorphes des sables littoraux (partie terrestre) de la Province du Natal (Rép. Sud Africaine) (Insecta: Collembola) Journal of African Zoology, Wavre, 111: 465-480.

Fernandes, L.H. \& M.C. Mendonça. 2002. Duas novas espécies de Pseudachorutinae (Collembola, Neanuridae) do Brasil. Boletim do Museu Nacional, Nova Série, Zoologia, Rio de Janeiro, 496: 1-8.

Fernandes, L. H. \& M. C. Mendonça. 2004. Collembola Poduromorpha do litoral de Maricá, Rio de Janeiro, Brasil. Revista Brasileira de Zoologia, Curitiba, 21 (1): 15-25.

Mendonça, C. de \& L.H. Fernandes. 1997. A new genus of Brachystomellinae from Brazil (Collembola: Neanuridae). Boletim do Museu Nacional, Nova Série, Zoologia, Rio de Janeiro, 379: 1-7.

Recebido em 01.III.2005; aceito em 11.X.2005. 